\title{
Estudo de parâmetros genéticos sobre o desempenho ponderal de bovinos da raça
}

\section{Caracu}

\author{
Study of genetic parameters on the weight performance of Caracu cattle \\ Estudio de parámetros genéticos sobre el rendimiento ponderal del ganado Caracu
}

Recebido: 25/01/2022 | Revisado: 02/02/2022 | Aceito: 09/02/2022 | Publicado: 14/02/2022

Flávio Luiz de Menezes

ORCID: https://orcid.org/0000-0002-4009-9338 Universidade Federal de Mato Grosso, Brasil

E-mail: flm.zootecnista@gmail.com

Fernanda Rodrigues Souza

ORCID: https://orcid.org/0000-0002-9606-3051 Universidade Federal de Mato Grosso, Brasil E-mail:nandaoliveirars2@gmail.com

Renata Rodrigues de Souza

ORCID: https://orcid.org/0000-0001-7624-7593 Universidade Federal de Mato Grosso, Brasil

E-mail: rodriguessouzarenata@gmail.com

Thauany Lucas de Souza Norberto

ORCID: https://orcid.org/0000-0002-3556-6878 Universidade Federal de Mato Grosso, Brasil E-mail: thauanynorberto2@gmail.com

Valdeci Alves De Rezende

ORCID: https://orcid.org/0000-0002-6220-6560 Universidade Federal de Mato Grosso, Brasil

E-mail:valdecialvesrezende@gmail.com

Cesar Enderle

ORCID: https://orcid.org/0000-0001-7765-4983 Universidade Federal de Mato Grosso, Brasil E-mail: cesarenderle29@gmail.com

Marcos Antonio Miotto de Souza ORCID: https://orcid.org/0000-0002-4790-3181 Universidade Federal de Mato Grosso, Brasil E-mail: marcosmiotto94@gmail.com

Clodoaldo Alves

ORCID: https://orcid.org/0000-0001-8453-0698 Universidade Federal de Mato Grosso, Brasil E-mail: clodoaldoalveszoo@gmail.com

Leonardo de Lima Silva

ORCID: https://orcid.org/0000-0002-2458-9150 Universidade Federal de Mato Grosso, Brasil E-mail: leokaroba@gmail.com

Cláudio Vieira de Araújo

ORCID: https://orcid.org/0000-0001-9378-7348 Universidade Federal de Mato Grosso, Brasil E-mail: cvaufmt@gmail.com

\section{Resumo}

Objetivou-se obter estimativas de herdabilidade e tendências genéticas de bovinos da raça Caracu sobre variáveis de pesos corporais. Os animais eram pertencentes ao estado de Mato Grosso, com informações sobre pesos nascimento, pesos ajustados para idades aos 240, 500, 830 dias de idade. Os grupos de contemporâneos foram formados pela combinação dos efeitos fixos de ano e época de pesagem e sexo do animal. Para a estimação dos componentes de variância foi utilizado modelos lineares misto com efeitos fixos de grupo de contemporâneos, efeitos aleatórios genético aditivo, genético materno (para os pesos pré desmama) e residual, utilizou o método da máxima verossimilhança restrita e as equações de modelos mistos para a predição de valores genéticos. As tendências genéticas foram estimadas por meio de regressão linear dos valores genéticos dos animais em função do ano de nascimento, para machos e fêmeas, separadamente. As estimativas de herdabilidades diretas foram de 0,24 a 0,45 para todos os pesos demonstrando a presença de variabilidade genética. As tendências genéticas foram estimadas para o peso nascimento de $-0,169$ e $-0,016$, peso a desmama de 0,117 e 0,138 , peso ao sobreano de 0,430 e 0,341 e peso adulto iguais a -0,541 e -0,176 quilogramas por ano, respectivamente, para machos e fêmeas. As herdabilidades e a 
tendência genética indicaram que diante a seleção direta para as características ponderais, obtém uma resposta esperada para a raça Caracu, denotando um aumento de velocidade de crescimento na recria e evidenciando a desmama como um critério de seleção.

Palavras-chave: Bovino de corte; Ganho genético; Produção animal; Seleção.

\begin{abstract}
The objective was to obtain estimates of heritability and genetic trends of Caracu cattle on body weight variables. The animals belonged to the state of Mato Grosso, with information on birth weights, age-adjusted weights at 240, 500, 830 days of age. Contemporary groups were formed by combining the fixed effects of year and time of weighing and animal sex. For the estimation of variance components, mixed linear models were used with fixed effects of contemporary groups, random effects, additive genetics, maternal genetics (for pre-weaning weights) and residual, using the restricted maximum likelihood method and the equations of mixed models. for the prediction of genetic values. Genetic trends were estimated through linear regression of the genetic values of the animals as a function of the year of birth, for males and females, separately. Direct heritability estimates ranged from 0.24 to 0.45 for all weights, demonstrating the presence of genetic variability. Genetic trends were estimated for birth weight of -0.169 and -0.016 , weaning weight of 0.117 and 0.138 , yearling weight of 0.430 and 0.341 and adult weight of -0.541 and 0.176 kilograms per year, respectively, for males and females. The heritabilities and the genetic tendency indicated that, in the face of direct selection for ponderal traits, an expected response for the Caracu breed was obtained, denoting an increase in growth speed in the rearing and evidencing weaning as a selection criterion.
\end{abstract}

Keywords: Beef cattle; Genetic gain; Animal production; Selection.

\title{
Resumen
}

El objetivo fue obtener estimaciones de la heredabilidad y tendencias genéticas del ganado Caracu sobre variables de peso corporal. Los animales eran pertenecientes al estado de Mato Grosso, con información de pesos al nacer, pesos ajustados para edades de 240, 500, 830 días de edad. Los grupos contemporáneos se formaron combinando dos efectos fijos de año y momento de pesaje y sexo del animal. Para una estimación de varianza de dos componentes para modelos lineales utilizados junto con efectos fijos de grupo contemporáneos, genética aditiva, genética materna (para pesos preponderados) y efectos aleatorios residuales, utilicé el método de máxima verosimilitud restringida y las ecuaciones de estos para una predicción de los valores genéticos. Como tendencias genéticas se estimaron mediante regresión lineal dos valores genéticos de dos animales en función del año de nacimiento, para machos y hembras, por separado. Como estimaciones de heredabilidad directa de 0,24 a 0,45 para todos los pesos, demostrando la presencia de carga genética. Las tendencias genéticas estimadas fueron para peso al nacer de -0.169 y -0.016 , peso al destete de 0.117 y 0.138 , sobrepeso al año de 0.430 y 0.341 y peso adulto igual a -0.541 y -0.176 kilogramos por año, respectivamente, para machos y hembras. Como las heredabilidades y una tendencia genética indican que ante una selección directa por caracteres de peso, obtenemos una respuesta esperada para la raza Caracu, denotando un aumento en la tasa de crecimiento en la creación y mostrando el destete como criterio de selección.

Palabras clave: Ganado vacuno; Ganancia genética; Producción animal; Selección.

\section{Introdução}

Em 2020 o Brasil possuía um rebanho de 187,55 milhões de cabeça de gado, com abate de 41,5 milhões de cabeças, considerado o maior rebanho bovino comercial do mundo, esta demonstra que o rebanho está estável, no mesmo ano abateu 43,3 milhões de cabeça e gerou cerca de 10,49 milhões TEC. Dessas, 26,07\% foram exportadas para cerca de 157 países, crescimento resultante do aumento na demanda de carne bovina pela China; e os outros 73,93\% foram destinados ao consumo interno com consumo per capita de $36,39 \mathrm{~kg} /$ ano. O impacto financeiro gerado pelo agronegócio da pecuária de corte foi de $10 \%$ do PIB total do Brasil (ABIEC, 2020).

Nesse contexto, a produção pecuária nos trópicos tem como pilar o uso de animais adaptáveis à suas condições edafoclimáticas, tornando os animais capazes de efetuar a termorregulação do seu metabolismo basal, fundamental para que os mesmos possam expressar desempenho desejável, no qual foram encontrados em várias regiões do Brasil (Pires et al. 2014).

No que diz respeito à adaptação, tem-se ferramentas que podem auxiliar, como a seleção associadas à ferramenta de cruzamento industrial, podendo assim acelerar o processo de adaptação dos animais, com os novos objetivos de seleção, tendo em vista a criação e utilização de novas raças como Senepol, Pitangueiras, e outros materiais genéticos, o benefício que foi gerado pela utilização do cruzamento industrial foi explorar os efeitos da heterose (Oliveira Filho, 2015). 
Assim sendo, o desempenho ponderal, comumente, está presente em sistemas de produção como um dos principais critérios de seleção na obtenção de aiment de produção em programas de seleção, sendo necessário a obtenção de parâmetros genéticos, bem como tendências genéticas, para tais critérios de forma a monitorar o desenvolvimento do programa de seleção.

Souza et al (2011), discutem que características de crescimento, como o peso corporal, medidas em todas as fases do desenvolvimento do animal, são importantes na determinação da eficiência econômica de qualquer sistema de produção de bovinos e podem ser recomendadas como critérios de seleção.

Nesse sentido, objetivou-se obter estimativas de herdabilidade e tendência genética do peso ao nascer, à desmama, sobreano e adulto em bovinos de corte da raça Caracu.

\section{Metodologia}

Utilizaram-se informações de 864 registros de pesos corporais ao nascimento (PN) e 768 registros de pesos padronizados aos 240 (P240), pesos corporais aos 500 dias (P500) e aos 830 (P830) dias de idade, de animais da raça Caracu pertencentes ao município de Sinop do estado do Mato Grosso/Brasil.

O sistema de produção da propriedade foi classificado por um regime alimentar a pasto, divididos em piquetes compostos por forrageiras da espécie Brachiaria brizantha cv. Xaraés. A matriz dos numeradores de parentesco continha 1789 animais, constituindo o arquivo de pedigree.

Os pesos foram ajustados (PC ajustado) para idades específicas (idade padrão) por meio da equação: $\mathrm{PC}$ ajustado=PCi + bi*(Id -idade padrão), em que bi é o coeficiente de regressão entre o peso corporal e idade dos animais (Id).

Foram compostos grupos de contemporâneos pela combinação dos efeitos fixos ano e época de pesagem e sexo. As épocas de pesagem foram compostas como: Época 1: de abril a setembro e Época 2, composta pelos demais meses do ano. Considerando-se apenas informações de grupo de contemporâneos com no mínimo três animais.

O modelo linear misto utilizado para a obtenção de componente de variância para os efeitos genético aditivo, e efeito residual, representado como: $\mathrm{y}=\mathrm{X} \beta+\mathrm{Z} \_1 \mathrm{a}+\mathrm{Z} \_2 \mathrm{~m}+\mathrm{e}$, em que: $y=$ vetor de observações, $\beta=$ vetor de efeitos fixos (grupo de contemporâneos), $a=$ vetor de efeito genético aditivo direto, $m=$ valor do efeito genético maternal (para pesos pré desmama), $e=$ vetor de efeito residual, $X=$ matriz de incidência que associa $\beta$ com $y, Z_{-} 1=$ matriz de incidência do efeito genético direto, $Z \_2$ = matriz de incidência do efeito genético maternal (para pesos pré desmama). Com a $\sim \mathrm{N}\left(0, A \sigma \_\mathrm{a}^{\wedge} 2\right)$ e e $\sim \mathrm{N}\left(0, \mathrm{I} \sigma \_\mathrm{e}^{\wedge} 2\right)$ em que $A$ é a matriz de parentesco.

Os componentes de covariância e herdabilidade foram estimados por meio do método da máxima verossimilhança restrita (REML) desenvolvido por Patterson and Thompson (1971) e as equações de modelos mistos (EMM) desenvolvido por Henderson (1973) para a predição de valores genéticos dos animais em cada característica.

As tendências genéticas foram estimadas regredindo o valor genético do animal em função do ano de nascimento do mesmo, utilizando-se o modelo $\mathrm{Yi}=\mathrm{b} 0+\mathrm{b} 1 \mathrm{Xi}+\mathcal{E}$, em que $=$ valor genético das características avaliadas do i ésimo ano de nascimento; $\mathrm{b} 0=$ intercepto; $\mathrm{b} 1=$ coeficiente angular da reta; $\mathrm{Xi}=\mathrm{i}$ ésimo ano de nascimento; ei = erro aleatório.

\section{Resultados}

Os resultados da análise descritiva para o PN foram, para a fêmea em média, $0,90 \mathrm{~kg}(2,55 \%)$ mais pesadas que os machos, evidenciando que nessa fase da vida dos indivíduos não há o dimorfismo sexual. Ao contrário do PN, ao P240 os machos apresentaram 13,67 kg (6,2\%) a mais que as fêmeas, denotando a diferenciação sexual aparente. Já em machos, em média $53 \mathrm{~kg}(14,75 \%)$ e $129 \mathrm{~kg}(21,8 \%)$ respectivamente mais pesados que as fêmeas, evidenciando que nessa fase da vida dos indivíduos não há destaque para o dimorfismo sexual (Tabela 1). 
Tabela 1 - Estatística descritiva com número de observações $(\mathrm{N})$, média e desvio-padrão fenotípico para os pesos corporais, em cada sexo e no geral.

\begin{tabular}{|c|c|c|c|c|}
\hline Machos & & $\mathrm{N}$ & Média & $\mathrm{DP}$ \\
\hline & PN & 462 & 34,45 & 9,46 \\
\hline & P240 & 377 & 220,35 & 42,93 \\
\hline & P500 & 228 & 358,72 & 86,28 \\
\hline & P830 & 108 & 493,13 & 85,72 \\
\hline \multicolumn{5}{|l|}{ Fêmeas } \\
\hline & PN & 402 & 35,35 & 4,64 \\
\hline & P240 & 391 & 206,68 & 40,03 \\
\hline & P500 & 157 & 305,79 & 76,48 \\
\hline & P830 & 100 & 463,92 & 83,92 \\
\hline \multicolumn{5}{|l|}{ Geral } \\
\hline & PN & 864 & 34,87 & 7,62 \\
\hline & P240 & 768 & 213,39 & 42,01 \\
\hline & P500 & 385 & 337,14 & 86,34 \\
\hline & P830 & 208 & 479,09 & 85,91 \\
\hline
\end{tabular}

Fonte: Autores.

As estimativas de herdabilidade encontradas denotaram que ao longo da vida a variância genética aditiva aumenta e o efeito genético materno diminui até o momento da desmama do animal, demonstrando a independência do desempenho no momento do desmame. Na estimativa de herdabilidade para os pesos pós desmame as magnitudes foram de moderadas a médias (Tabela 2.).

Tabela 2 - Estimativas de componentes de variância e herdabilidade para PN, P240, P500 e P830.

\begin{tabular}{lcccc}
\hline \multicolumn{1}{r}{ Componente de variância } & PN & P240 & P500 & P830 \\
\hline Materno & 0,5077 & 26,670 & - & - \\
Aditivo & 1,669 & 665,000 & 252,30 & 1189,00 \\
Ambiental & 4,542 & 789,700 & 790,30 & 2431,00 \\
\hline Herdabilidade Direta & 0,25 & 0,45 & 0,24 & 0,33 \\
\hline Herdabilidade Materna & 0,08 & 0,01 & - & - \\
\hline
\end{tabular}

Fonte: Autores.

No período de 15 anos estudados a mudança genética anual de bovino Caracu foi estimado a tendência genética para o P240, e P500 dias, no qual tiveram um incremento para macho e fêmea (Figura 3 e Figura 2), entretanto, para o PN e P830, houveram repressão no peso corporal para machos e fêmeas (Figura 1 e Figura 4). 
Figura 1 - (A) - Tendência genética para o PN em machos (y = -0,0169x - 0,0259) e (B)Tendência genética para o PN em fêmeas $(y=-0,0163 x+0,0321)$. Obs= regressão dos valores genéticos médios sobre o ano de nascimento de cada grupo de contemporâneos e Pred= regressão dos valores médios genéticos sobre o ano de nascimento de cada grupo de contemporâneos;

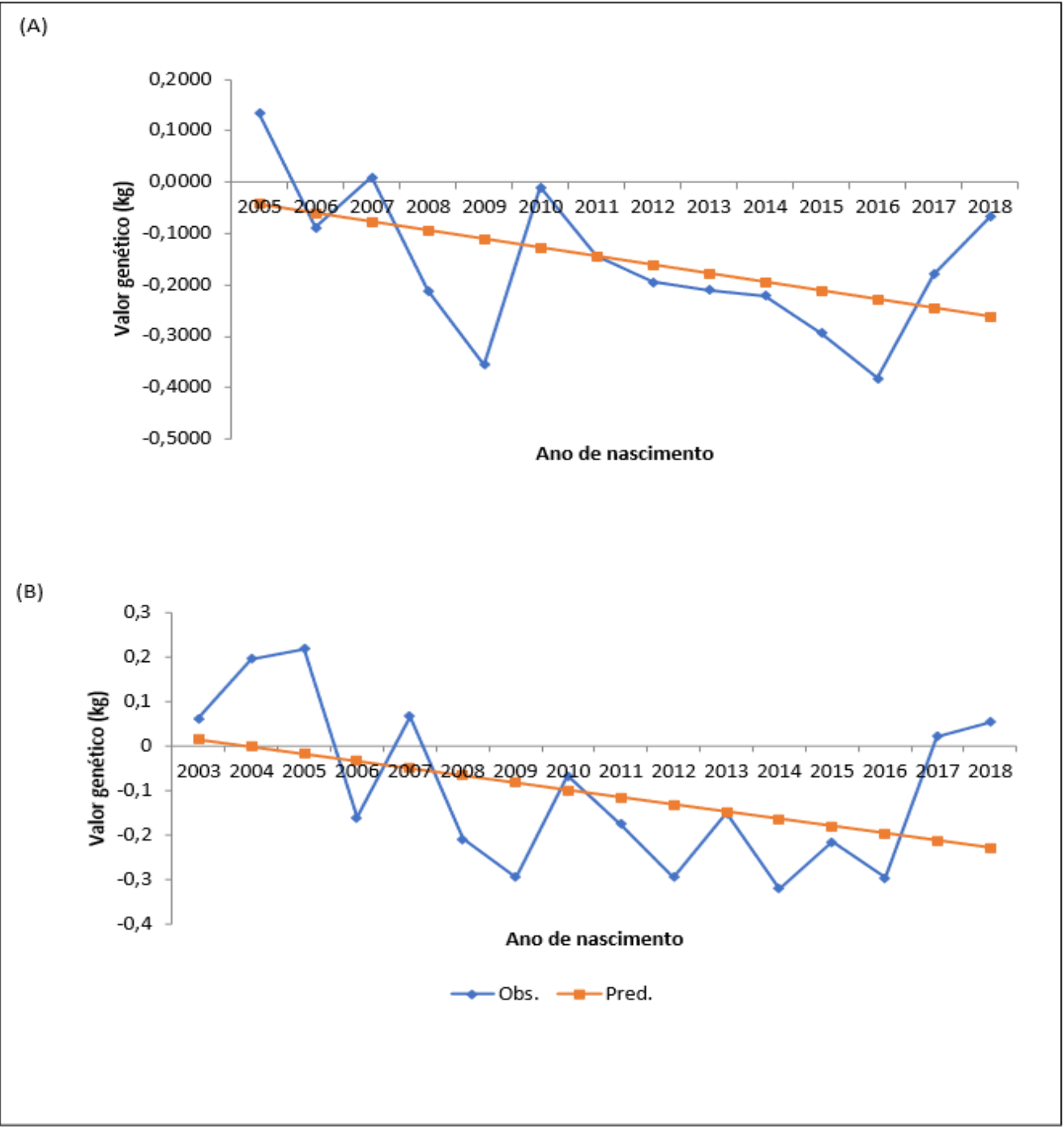

Fonte: Autores. 
Figura 2 - (A)Tendência genética para o P240 em machos $(y=0,1167 x+0,6735)$ e (B) Tendência genética para o P240 em fêmeas $(y=0,2158 x-0,213)$. Obs= regressão dos valores genéticos médios sobre o ano de nascimento de cada grupo de contemporâneos e Pred= regressão dos valores médios genéticos sobre o ano de nascimento de cada grupo de contemporâneos;

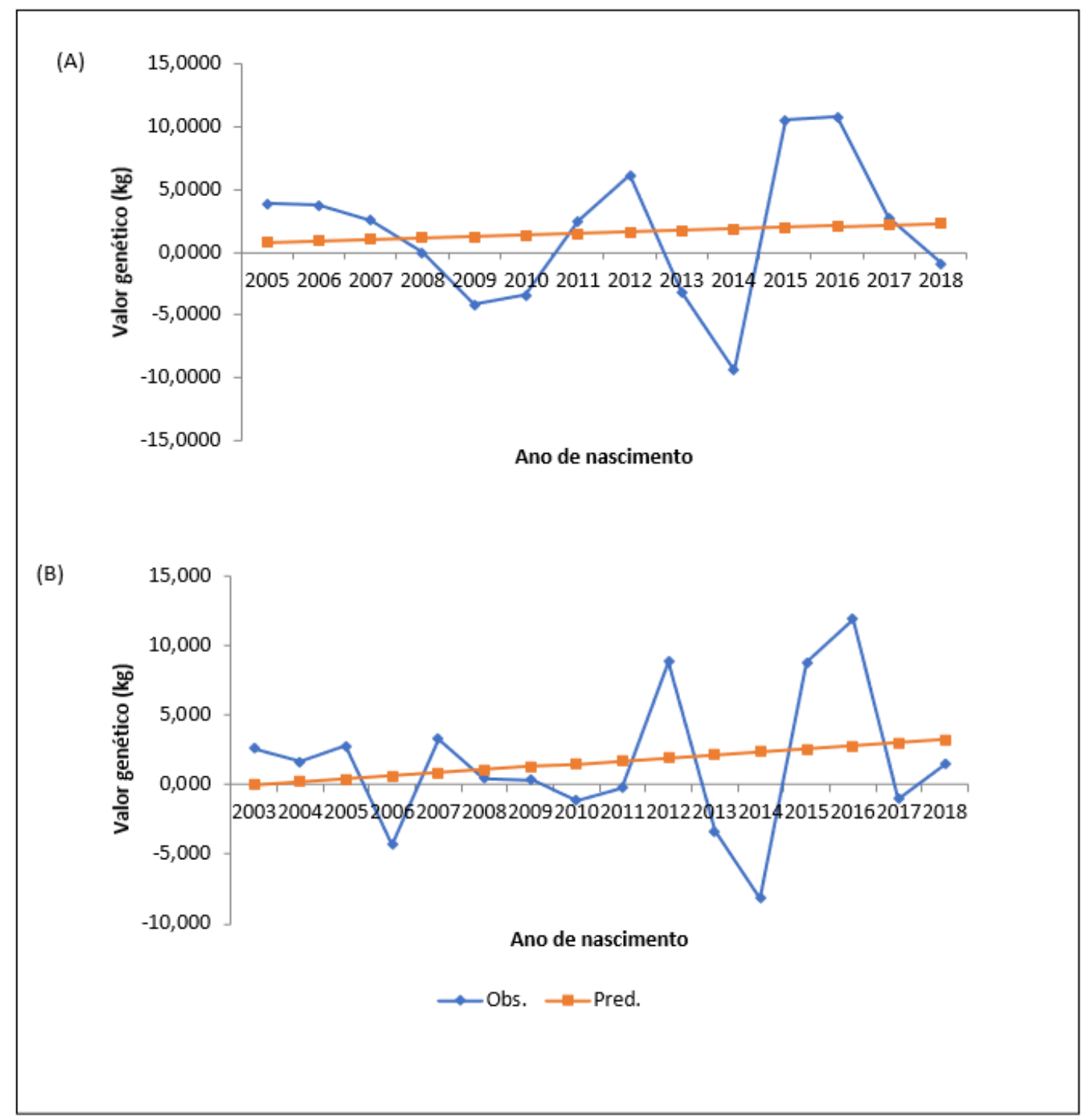

Fonte: Autores. 
Figura 3 - (A) Tendência genética para o P500 em machos (y =0,4299x - 2,3727), (B) Tendência genética para o P500 em fêmeas $(y=0,2978 x-0,9627)$ Obs= regressão dos valores genéticos médios sobre o ano de nascimento de cada grupo de contemporâneos e Pred= regressão dos valores médios genéticos sobre o ano de nascimento de cada grupo de contemporâneos;

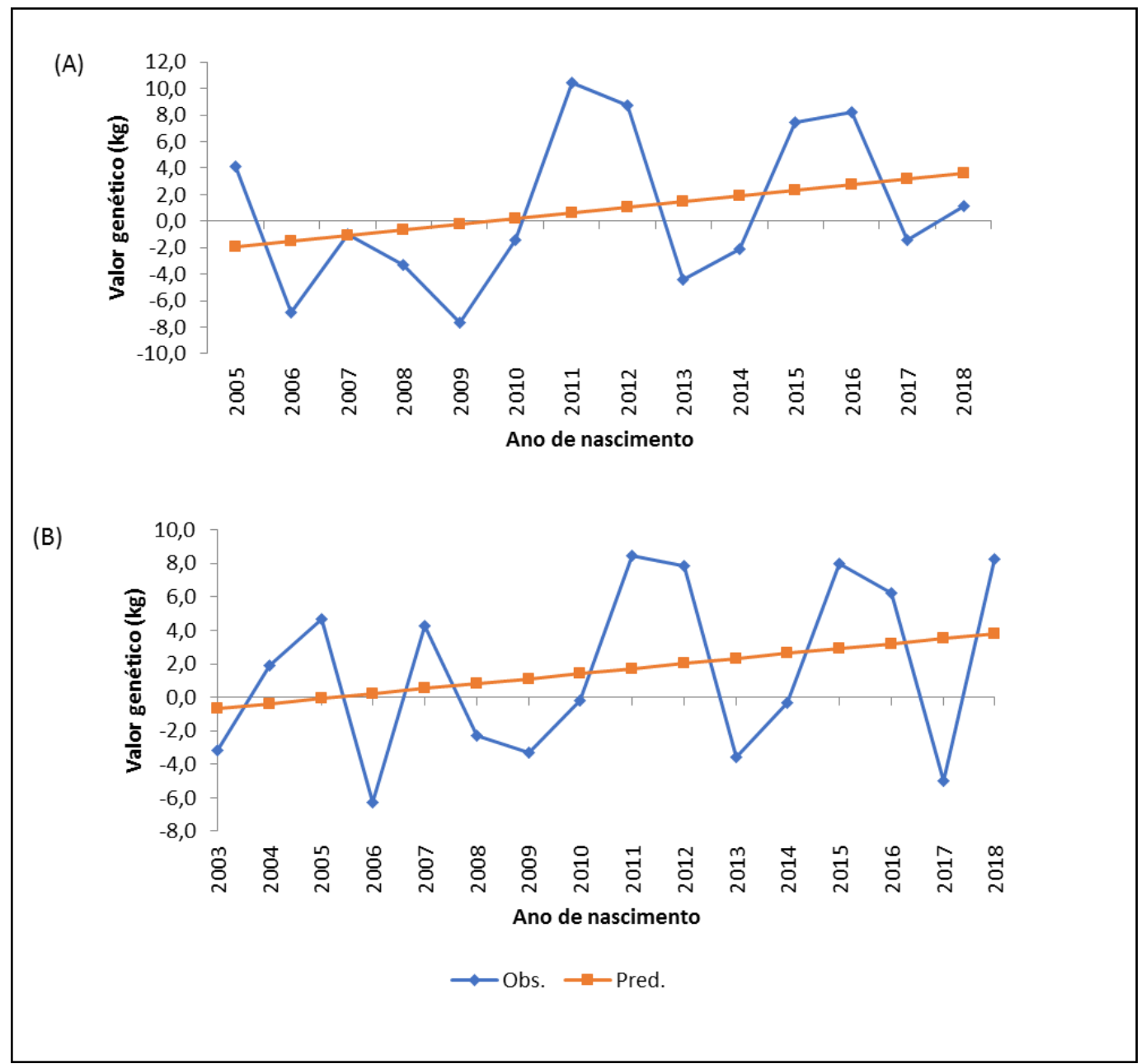

Fonte: Autores. 
Figura 4 - Tendência genética para o P830 em machos $(y=-0,5417 x+4,518)$; Tendência genética para o P830 em fêmeas ( $\mathrm{y}=-0,2429 \mathrm{x}+2,5636)$ ) Obs= regressão dos valores genéticos médios sobre o ano de nascimento de cada grupo de contemporâneos e Pred= regressão dos valores médios genéticos sobre o ano de nascimento de cada grupo de contemporâneos;

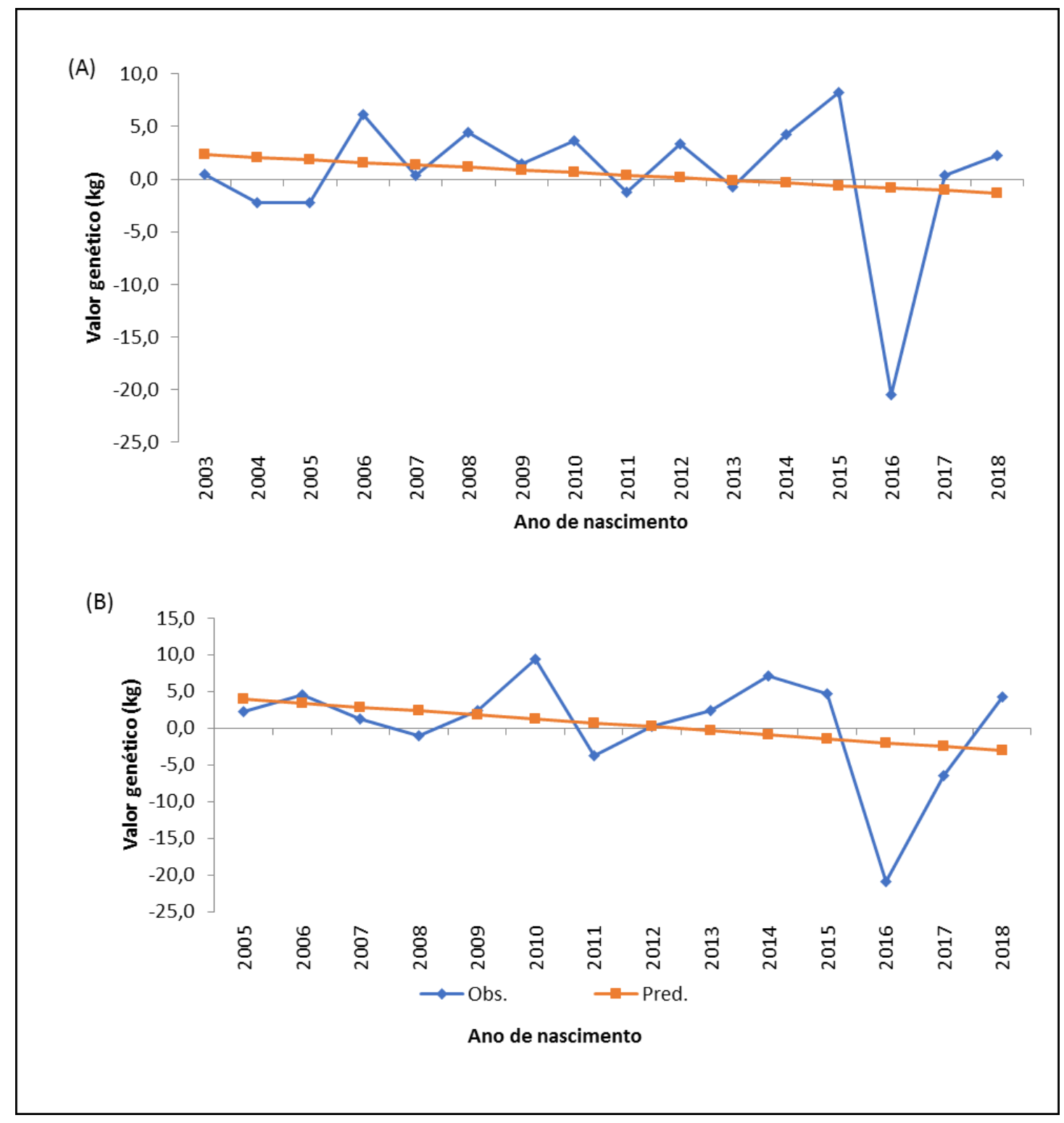

Fonte: Autores.

\section{Discussão}

As estimativas de herdabilidade direta e materna encontradas para peso ao nascimento, foram semelhantes aos obtidos

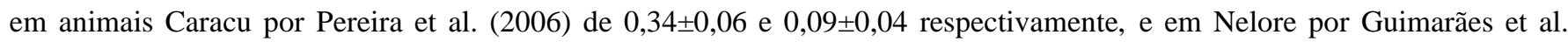
(2017) de 0,27, demonstrando a similaridade entre raças, em detrimento do peso ao nascimento ter pouca variabilidade na espécie, portanto, a possibilidade da característica ser utilizada como critério de seleção em avaliações genéticas.

Os valores obtidos para o peso a desmama por Evangelista et al (2020) e Amaral (2014) foram inferiores ao estudo de $168,90 \pm 32,14$ e 168,8 $\pm 28,4 \mathrm{~kg}$ respectivamente, para peso padronizado aos 205 dias de idade em animais Nelore. Observouse estimativas de herdabilidade direta, no modelo animal, superiores à 0,08 a 0,34 para peso ao desmame por Ambrosini et al. (2014b; 2016) e malhado (2005). Ja Souza et al (2011) estimaram peso desmama de 180,5 $\pm 36,3$, denotando que o desempenho 
de animais Caracu no momento da desmama foram superiores possivelmente pela habilidade materna encontrada.

Valores obtidos de peso ao desmame para animais Nelore por Amaral (2014) e Malhado et al. (2005) de 0,12 a 0,20, demonstram que em animais Caracu seu peso no momento da desmama sobressaíram às demais raças, evidenciando deste modo a relação com animais mais adaptados, bem como a habilidade materna, responsável por grande parte de seu crescimento neste período.

A herdabilidade materna para P205 encontradas em animais Caracu segundo Malhado et al. (2005) Souza et al. (2011) foram iguais a $0,12 \pm 0,02$ e 0,05 $\pm 0,01$ respectivamente, demonstrando que a herdabilidade materna para o estudo nos animais da raça Caracu foram superiores encontradas por Ambrosini et al. (2016) e (2014b) com valores de 0,03 à 0,19.

Para o peso padronizado aos 240 dias o incremento em $\mathrm{kg} / \mathrm{ano}$ de fêmeas foi superior ao observado em machos, evidenciando que responderam substancialmente a seleção aplicada (Figura 2.). E, quando comparado a animais Nelore Mocho oriundos da região Nordeste demonstrado por Amaral et al. (2014) tem-se acréscimo de 0,016 kg/ano, enquanto Oliveira (2017) encontrou um ganho genético de 0,1870 kg/ano, evidenciando um aumento de peso ao desmame ao longo dos anos, com valores superiores a estimativas encontradas em animais Caracu.

Quintino et al. (2004) e Pereira et al. (2008) estimaram valores com tendência genética em animais Caracu sobre os pesos ao nascimento e a desmama, respectivamente de, 0,0654 e 0,08 \pm 0,02, 0,6226 e 0,48 $\pm 0,11 \mathrm{Kg}$ /ano, denotando poucas alterações anuais ao nascimento semelhante encontrado no trabalho (Figura 1.) e altos ganhos a desmama superior encontrado no estudo que foi positivo, mas com uma magnitude moderada de ganho anual.

Como critério de seleção o peso 240 dias é de suma importância para o sistema de cria de bovinos, podendo ser utilizado no momento de descarte e seleção de animais, permitindo eleger animais superiores ao sistema de produção nos trópicos.

O peso corporal médio para os animais Caracu aos 500 dias de idades P500 (Tabela 2.), foi superior ao peso encontrado por Moreira et al (2016) e Pereira et al (2008), que foram de respectivamente, $287 \mathrm{~kg}$ e 281,1 kg. Mercadante (2005), encontrou para machos e fêmeas caracu, peso sobreano de respectivamente, 329,31 $\pm 60,57$ e 253,65 $\pm 46,83$, assim como Pereira et al (2008), que obtiveram valor de 281,1 kg para fêmeas Caracu, demonstrando que os animais em questão tornaram mais pesados. Amaral et al (2014), e Laureano et al (2011), obtiveram os valores a cerca de 274,72 a 307,25 $\pm 62,59$ $\mathrm{kg}$, para peso sobreano para animais Nelore.

As estimativas de herdabilidade para P500 (Tabela 2), foram semelhantes às descritas por Lira et al, (2013) de 0,24

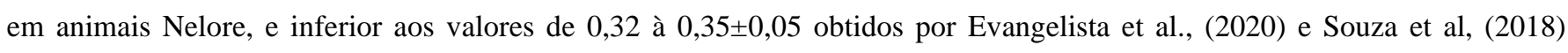
respectivamente, também para a raça Nelore. Os dados da (Tabela 2) para herdabilidade aos P500 dias possuem valores de moderados a média, o que torna a seleção para a característica um mecanismo efetivo de melhoramento.

As estimativas de herdabilidade para peso sobreano da raça Nelore Amaral et al (2014), obteve herdabilidade para P550 de 0,18 $\pm 0,03$, semelhante ao obtido por Laureano et al (2011) de 0,24 $\pm 0,02$, diferindo do encontrado por Santos et al (2012), que obteve herdabilidade de 0,41 para P550, essa discrepância entre valores obtidos pode ter ocorrido decorrente da população avaliada por ambos, sendo de 128.148 e 45.388 animais respectivamente. Quando comparado a herdabilidade de 0,24 ao P500 da raça Caracu, nos demonstra a maior variabilidade genética da raça, procedendo assim ganhos genéticos mais expressivos.

Para a tendência genética sobre peso corporal aos 500 dias de idade, sobressaíram com 0,132 kg/ano em relação às fêmeas (Figura 3.), indicando uma aceleração de deposição de tecido muscular em machos.

A tendência genética para peso aos 550 dias obtidas na literatura por Souza et al (2011) na raça Nelore, demonstraram ganhos genéticos de 0,946 kg/ano, destacando a velocidade de deposição tecidual desses animais. Já Souza et al (2018), também avaliaram peso ao sobreano de animais Nelore e obtiveram ganho anual de 0,506 kg/ano equivalente ao identificado 
nos animais Caracu; e no mesmo trabalho entre suas características, deste modo, a característica como critério seletivo adotado em programas de seleção contribui com a evolução do rebanho.

A média para P830 foi próximo ao valor encontrado por Moreira et al (2016), com peso idade adulta de $474 \mathrm{~kg}$ em animais caracu (790 dias), já Mello et al (2006), encontraram para vacas da raça Canchim, peso médio a idade adulta de 500 $\mathrm{kg}$, explicado pela origem das raças e enfocando que as fêmeas da raça Caracu tornaram mais compactos.

A estimativa de herdabilidade para P830, indicou a viabilidade da seleção direta como critério para bovinos da raça Caracu, este valor foi similar ao encontrado de 0,26 (Rosa et al 2001) em bovinos Nelore, entretanto foi inferior encontrado de 0,43 (Pedrosa et al 2010), a diferença encontrada pelos outros autores sugerem que na raça Nelore temos uma maior variabilidade fenotípica, porém em ambos é abordado a importância da mesma para o monitoramento do peso adulto da raça e do próprio rebanho.

A tendência genética para peso padronizado aos 830 dias, houve repressão no peso corporal adulto para ambos os sexos (Figura 4), isto representou um peso assintótico e um tamanho corporal inferior aos animais contemporâneos relacionados a animais mais compactos.

\section{Conclusão}

Características de desempenho ponderal apresentam com variabilidade genética aditiva suficiente para melhorias no desempenho do rebanho, destacando o período de avaliação da idade ao desmame ao soberano. As tendências genéticas indicaram que o programa de seleção genética aplicado sobre os animais, conduziram os mesmos a um biótipo compactos e precoces.

Para trabalhos futuros com a raça Caracu, sugere se haver mais avaliações genéticas relacionadas ao desempenho ponderal visando melhoria genética da raça para um sistema de produção de carne com foco em Precocidade e velocidade de crescimento.

\section{Referências}

ABIEC. (2020). Associação Brasileira das Indústrias Exportadoras de carne. Perfil da Pecuária no Brasil. http://abiec.com.br/publicacoes/beef-report-2020/.

Amaral, R. D. S., Carneiro, P. L. S., Martins Filho, R., Ambrosini, D. P., \& Malhado, C. H. M. (2014). Tendências, parâmetros fenotípicos e genéticos de características de crescimento em bovinos Nelore mocho do Nordeste brasileiro. Revista Brasileira de Saúde e Produção Animal, 15(2), 261-271.

Ambrosini, D. P., Malhado, C. H. M., Braccini Neto, J., Martins Filho, R., Mello Affonso, P. R. A. D., \& Carneiro, P. L. S. (2014). Reaction norms of direct and maternal effects for weight at 205 days in Polled Nellore cattle in north-eastern Brazil. Archives Animal Breeding, 57(1), 1-11.

Ambrosini, D. P., Malhado, C. H. M., Martins Filho, R., \& Carneiro, P. L. S. (2016). Interação genótipo x ambiente via modelos de normas de reação para características de crescimento em bovinos Nelore. Pesquisa Agropecuária Brasileira. 51(2), 177-186.

Evangelista, A. F., Cavalcante, D. H., Malhado, C. H. M., Campelo, J. E. G., Carvalho, G., \& de Sousa Júnior, S. C. (2020). Estimação de parâmetros genéticos para características de crescimento em bovinos Nelore Mocho da Região Norte do Brasil. Embrapa Meio-Norte-Artigo em periódico indexado (ALICE).

Guimarães, N. C., Viu, M. A. O., Magnabosco, C. U. M., Lopes, F. B.,Ferraz H. T.,Ferraz H. T. \& Fernandes S. T. (2017) Parâmetros genéticos de caracteres de crescimento e carcaça em rebanhos selecionados da raça nelore. In: Congresso Brasileiro De Zootecnia Zootec, Santos.

Henderson, C. R (1973). Avaliação de touros e tendências genéticas. Journal of Animal Science, 1973 (Simpósio). $10-41$.

Laureano, M. M. M., Boligon, A. A., Costa, R. B., Forni, S., Severo, J. L. P., \& Albuquerque, L. G. D. (2011). Estimativas de herdabilidade e tendências genéticas para características de crescimento e reprodutivas em bovinos da raça Nelore. Arquivo Brasileiro de Medicina Veterinária e Zootecnia. 63(1), 143152 .

Lira, T. S., Alexandrino, E., Lopes, F. B., Nepomuceno, L. L., de Sousa Pereira, L., Lôbo, R. B., \& Ferreira, J. L. (2013). Interação genótipo-ambiente em pesos pós-desmama de bovinos Nelore criados nos estados do Maranhão, Mato Grosso e Pará. Acta Veterinária Brasilica. 7(4), $282-287$.

Malhado, C. H. M., Martins Filho, R., Lôbo, R. N. B., Facó, O., Azevedo, D. M. M. R., Souza, J. C. D., \& Oliveira, S. M. P. (2005). Tendências genéticas para características relacionadas à velocidade de crescimento em bovinos Nelore na região Nordeste do Brasil. Revista Brasileira de Zootecnia. 34(1), 60-65. 
Mello, S. D. P., Alencar, M. M. D., Toral, F. L. B., \& Gianlorenço, V. K. (2006). Estimativas de parâmetros genéticos para características de crescimento e produtividade em vacas da raça Canchim, utilizando-se inferência bayesiana. Revista Brasileira de Zootecnia. 35(1), 92-97.

Mercadante, M. E. Z. (2005). Caracu, O Bos taurus brasileiro adaptado aos trópicos: experiências de um programa de seleção para peso ao sobreano. Agrociencia-Sitio en Reparación. 9(1-2), 485-494.

Moreira, R. P., Mercadante, M. E. Z., Pedrosa, V. B., Cyrillo, J. N. D. S. G., \& Henrique, W. (2016). Growth curves on females of the Caracu breed. Semina: Ciências Agrárias. 37(4), 2749-2757.

Oliveira Filho, A. (2015). Produção e Manejo de Bovinos de Corte./Cuiabá-MT: KCM Editora,155p.; (versão - ebook)ISBN 978-85-7769-212-5. https://acrimat.org.br/portal/wp-content/uploads/2017/05/livro-producao-e-manejo-de-gado-de-corte.pdf

Oliveira, M.H.V. (2017). Estimativas de tendências e parâmetros genéticos e fenotípicos para escore visual de úbere, habilidade materna e peso à desmama em bovinos da raça Nelore. 47f. Trabalho de Conclusão de Curso (Graduação) - Universidade Federal de São João Del Rei, São João Del-Rei.

Patterson, HD, \& Thompson, R. (1971). Recuperação de informações entre blocos quando os tamanhos dos blocos são desiguais. Biometrika. 58(3), 545-554.

Pedrosa, V. B., Eler, J. P., Ferraz, J. B. S., de Vasconcelos Silva, J. A. I., Ribeiro, S., Silva, M. R., \& Pinto, L. F. B. (2010). Parâmetros genéticos do peso adulto e características de desenvolvimento ponderal na raça Nelore. Revista Brasileira de Saúde e Produção Animal. 11(1), 104-113.

Pereira, M. C., Mercadante, M. E. Z., Albuquerque, L. G. D., Razook, A. G., \& Figueiredo, L. A. D. (2006). Estimativas de parâmetros genéticos de características de crescimento em um rebanho Caracu selecionado para peso ao sobreano. Revista Brasileira de Zootecnia. 35(4), $1669-1676$.

Pereira, M. C., Mercadante, M. E. Z., Razook, A. G., Figueiredo, L. A., \& Albuquerque, L. G. D. (2008). Results of 23 years of selection for post-weaning weight in a Caracu beef herd. South African Journal of Animal Science. 38(2), 136-144.

Pires, R. M. L., Alvarez, R. H., do Amaral, J. B., Monteiro, F. M., de Melo, A. J. F., Trevisol, E., \& Lara, M. A. C. (2014). Caracterização citogenética das raças bovinas Caracu, Junqueira, Pantaneira e Patuá. Boletim de Indústria Animal. 71(4), 332-340.

Quintino, H. P., Ferraz, J. B. S., \& Eler, J. P. (2004). ESTUDO DA TENDÊNCIA GENÉTICA E FENOTÍPICA PARA BOVINOS DA RAÇA CARACU. In : V Simpósio da Sociedade Brasileira de Melhoramento Animal SBMA . Pirassununga http://www.sbmaonline.org.br/anais/v/trabalhos/pdfs/bc026.pdf.

Rosa, A. D. N., Lôbo, R. B., Oliveira, H. N. D., Bezerra, L. A. F., \& Reyes Borjas, A. D. L. (2001). Peso adulto de matrizes em rebanhos de seleção da raça Nelore no Brasil. Revista Brasileira de Zootecnia. 30(3), 1027-1036.

Santos, L. H., Oliveira, S. M. P. D., Malhado, C. H. M., Carneiro, P. L. D. S., Martins Filho, R., Lôbo, R. N. B., \& Rodrigues, D. D. S. (2012). Estrutura populacional e tendências genéticas e fenotípicas da raça Guzerá no Nordeste do Brasil. Revista Brasileira de Saúde e Produção Animal. 13(4), $1032-1043$.

Souza, J. C., Silva, L. O. C., Gondo, A., Freitas, J.A., Malhado, C. H. M., Filho, P. B. F., Sereno, J. R. B., Weaber, R. L., \& Lamberson, W. R. (2011). Parâmetros e tendências genéticos de peso de bovinos criados à pasto no Brasil. Archivos de Zootecnia. 60(231), 457-456.

Souza, J. C., de Resende, M. P. G., da Silva, L. O. C., Gusmão, M., Gondo, A., Mota, M. F., Freitas J. A., \& Silva, R. M. (2018). Parâmetros e tendências genéticas para peso ao sobreano de animais Nelore criados no estado do Paraná, Brasil. Revista Colombiana de Ciencia Animal-RECIA. 10(1), 68-73. 